\title{
Dos teorías informacionales de la conciencia
}

Diego Llontop

Universidad San Ignacio de Loyola

Lima, Perú

\author{
Recibido: 7 de mayo del 2017 / Aceptado: 15 de junio del 2017 \\ doi: https://doi.org/10.26439/persona2017.n20.1744
}

El artículo presenta dos teorías que buscan explicar la conciencia sobre la base del concepto de información. Ortiz entiende la información como una estructura material que configura el neocórtex humano, y de esta forma constituye la conciencia, mientras que Chalmers considera la información como un "espacio abstracto", no material, pero realizable en lo físico, por tanto, un elemento que podría ayudar a explicar el carácter inefable de la conciencia. Ambas perspectivas aparecen en un contexto teórico en el cual el materialismo clásico como hegemónica imagen científica del mundo muestra una aparente insuficiencia para dar cuenta del aspecto fenoménico de la conciencia.

conciencia / información / materialismo

\section{Two Informational Theories of Consciousness}

The paper presents two theories that seek to explain consciousness on the basis of the concept of information. Ortiz understands information as a material structure that shapes the human cerebral cortex, thus configuring consciousness, while Chalmers considers information as an "abstract space" not material but realizable in the physical, therefore, an element that could explain the ineffable character of consciousness. Both perspectives appear in a theoretical context in which classical materialism as hegemonic scientific image of the world shows an apparent insufficiency to account for the phenomenological aspect of consciousness.

consciousness / information / materialism 


\section{EL PROBLEMA DE LA CONCIENCIA}

Intente por un momento imaginarse sin conciencia. ¿Puede? El reto es insuperable y hasta podríamos decir paradójico. Si pudiera, eso implicaría que podría pensar en que no está pensando. En otras palabras, el hecho de poder imaginarse sin conciencia impediría que pueda tener una idea siquiera aproximada de lo que sería no tenerla. Ambas opciones no pueden ir juntas. O pierde la conciencia y, por lo tanto, no puede generar ningún contenido; o se mantiene consciente, por tanto, todos los contenidos de conciencia posibles que pudiera producir negarían el objetivo imposible de acercarse siquiera a imaginar cómo sería no tenerla.

Lo que he sostenido en el párrafo precedente es una simplificación del problema con el objetivo de llamar su atención. Y es que cuando hablamos de conciencia hay diversas acepciones a las que uno se puede referir. Por ejemplo, David Chalmers (1995) identifica varios sentidos: desde la conciencia de experiencias sensoriales, la conciencia de imágenes o imaginería mental, el pensamiento consciente, la experiencia de las emociones y, por último, la noción del Yo o Self. Sin embargo, considero que todas estas posibles acepciones implican necesariamente una noción general que incluye una suerte de "experiencia fenoménica central", en la cual y desde la cual experimento efectiva y actualmente el mundo; en la cual tengo la plena certeza de la vivencia, me encuentro en ella, sin posibilidades de disociarme de la misma, como veíamos al inicio.
Siewert (2014) llama la atención sobre este aspecto, conocido como experiencia subjetiva o en lenguaje más técnico conciencia fenoménica:

[L]a conciencia está envuelta de manera penetrante tanto en la percepción como en el pensamiento [...] es aquello a través de lo cual sabemos lo que está a nuestro alrededor, lo que está en nuestras mentes, y lo que significamos; es aquello por lo cual en primer lugar tenemos mentes; descansa en el centro de nuestra preocupación por nosotros mismos y por los demás. Si eso no la hace importante, ¿qué lo haría? (p. 200)

Consideramos que esta suerte de sentido fundamental que es la conciencia no puede ser ignorado, como pretenden algunos en el ámbito de la filosofía de la mente. Lo problemático de una maniobra teórica eliminativa de la conciencia queda en evidencia al remitirnos al carácter central o inevitable del fenómeno. Podemos imaginarnos sin cualquiera de los sentidos. Pero no podemos imaginarnos sin sentido. El acto de imaginar o de pensar ya implica un sentido. Nuestra noción de sentido supone una determinada relación entre el pensamiento y lo pensado. Una necesaria interdependencia entre lo uno y lo otro. A esto hacía referencia el viejo Parménides cuando decía que "el ser es y el no ser no es". Una de las razones que esboza para defender dicha conclusión es que el no ser ni siquiera puede ser pensado. Es decir, para el filósofo griego lo único que puede haber es lo pensado. Y lo no pensado no puede ser justamente porque no sería en el sentido de presencia consciente actual. 
¿Qué evidencia podríamos tener de lo que es fuera de la conciencia? Lo único que es, es lo pensado, pues lo que no es, ni siquiera se puede pensar. Una versión derivada de esta reflexión originaria la encontramos en Gabriel (2016) y su tesis de la ineluctabilidad: "Nuestra posición subjetiva sigue siendo ineluctable incluso para la mejor ciencia del futuro, ya que a partir de ella estamos en condiciones de desarrollar el ideal de objetividad absoluta" (p. 127).

Algunos dirían que este camino nos llevaría peligrosamente a la orilla del idealismo. Creemos que esto no es necesariamente cierto. Por otrolado, resulta interesante comprobar que en las últimas décadas el muy duradero monopolio cognoscitivo del viejo materialismo ha empezado a ponerse en cuestión. Diríamos, incluso, a fracturarse. Esta vulnerabilidad podría explicarse por el dogmatismo ideológico, que se sedimentó como una reacción comprensible frente a la explicación profundamente especulativa del escolasticismo medieval, junto con el ímpetu experimental galileano del siglo XVI y la posterior síntesis newtoniana en el siglo XVIII, generadora de innegables éxitos explicativos y, por lo mismo, predictivos. Estos hechos promueven una hegemonía empirista en filosofía de la ciencia, que, sin embargo, no tarda en ponerse en cuestión desde diversos frentes. Solo por mencionar dos: Kuhn (2006) con su idea de paradigma, que muestra que la labor científica no es puramente empírica, sino que se encuentra influida por los acuerdos de la comunidad científica; y Popper
(1994) con su racionalismo crítico, de corte deductivo, y su consecuente cuestionamiento de la inducción, de corte empírico, como supuesto fundamento metodológico de la ciencia. Estas dos perspectivas abrirían el camino, en la segunda mitad del siglo $\mathrm{xx}$, a una mirada más sofisticada de la ciencia, en la cual las restricciones del empirismo no ejercen el monopolio cognoscitivo.

Por otro lado, en el ámbito de la teoría de la mente, han sido muy influyentes las críticas de Putnam (1981) al materialismo, correspondientes con la afirmación de que un atributo psicológico no es lo mismo que un atributo físico. Este giro funcionalista, conocido como realizabilidad múltiple, implicó alejarse del reduccionismo de la teoría de la identidad entre la mente y el cerebro, asumiendo que las propiedades mentales no tienen que estar asociadas necesariamente al cerebro humano. Posteriormente, Putnam (1994) rompe definitivamente con la mirada funcionalista que él mismo se había encargado de defender, en el sentido de negar que fuese posible entender las propiedades mentales de una vez por todas, ya que, a pesar de no corresponder necesariamente con la materia cerebral, estas propiedades no podrían estar disociadas de la cultura y la historia humana. En consecuencia, no se les podría entender como atributos inherentes a la interioridad del individuo visto de forma aislada.

En la misma línea y adelantando las descripciones que realizaremos de las dos posturas teóricas objeto de este estudio, mencionamos, por un lado, a Chalmers (1995) y su dualismo naturalista, que a 
su vez se apoya en la crítica de Jackson (1982) al materialismo. Chalmers considera que abandonar el monismo materialista no es necesariamente caer en el idealismo o en el misterio, pues puede ser pensable una especie de dualismo de propiedades, reconociendo (con Jackson) que las propiedades físicas no pueden dar cuenta por sí mismas de las experiencias subjetivas. Esta actitud de apelar a explicaciones más allá del paradigma materialista se refleja también en el rescate de una posición ontológica del siglo xx, ignorada por muchos años, pero ahora rescatada en vista de la insuficiencia explicativa del materialismo con relación al problema de la conciencia. Nos referimos al monismo neutral de Bertrand Russell, consistente en asumir que el universo podría tener propiedades básicas que no conocemos, que podrían ser el fundamento de los aspectos mental y físico que podemos percibir. Se trataría de protopropiedades (protofísicas y protomentales) que "podrían establecer el puente sobre el vacío explicativo entre mente y materia y asegurarían la eficiencia causal de la mente junto a la materia" (Kriegel, 2014, p. 4). El hecho de formular hipótesis sobre propiedades protofísicas implicaría necesariamente ubicarse por detrás de una metafísica únicamente material. Esto no llevaría necesariamente a pensar en propiedades sobrenaturales, con lo cual el naturalismo de las propuestas se preservaría.

Como podemos ver, el materialismo radical está siendo reubicado con respecto a otras perspectivas posibles, en pos de una explicación viable de la vida consciente. Podríamos considerar el problema de la conciencia como el motor cognitivo de esta reubicación teórica. Inicialmente, en la segunda mitad del siglo $\mathrm{xx}$ se inicia la crítica al modelo empírico radical del positivismo lógico. Luego, de la mano de la filosofía de la mente, se pone en duda la hegemonía materialista para explicar las funciones conscientes. Tanto el reduccionismo como el eliminativismo ya no aparecen como las únicas vías posibles de solución del problema mente-cuerpo, y se abren paso nuevos esfuerzos en pos de un cuadro científico que incluya a la conciencia dentro de sus programas de investigación. Que la misma noción de ciencia haya experimentado una mayor sofisticación implica también el cuestionamiento de las explicaciones mecanicistas y, por lo mismo, la búsqueda de un principio causal alternativo al de los engranajes del reloj, en el que cada movimiento mecánico es causa única, suficiente y determinista de todo el circuito causal posterior, en una secuencia lineal en una sola dirección. Como veremos, la teoría informacional de Ortiz (2008) descansa en un principio fundamental de naturaleza teleológica, para el cual el desarrollo de la vida no es mecánico, sino dialéctico. Los estadios posteriores de un determinado desarrollo influyen en los precedentes de tal forma que los transforman, convirtiéndolos en un soporte activo de todo el sistema. En esta lógica no determinista descansa su propuesta teórica sobre la conciencia, a nuestro entender, un reflejo de los nuevos aires metafísicos en la ciencia de nuestros tiempos. 
El problema de la conciencia implica una dificultad central. Lo que Chalmers cataloga como el "problema difícil de la conciencia" consiste en que no tenemos idea de cómo un sistema físico como el cerebro puede generar la experiencia subjetiva. ¿Cómo surge la conciencia? ¿Cuál es la relación entre cerebro y conciencia? ¿Por qué existe la conciencia? ¿Por qué debe haber algo que sea eso que es "ser como algo"? ¿Esa vivencia subjetiva de la primera persona? Las dos teorías que analizaremos a continuación proponen caminos posibles para la solución de algunos de estos problemas basándose en el concepto de información: Ortiz, desde el ámbito de las neurociencias, y Chalmers, desde el ámbito de la filosofía analítica. Intentaremos evaluarlas, compararlas $\mathrm{y}$ ver hasta qué punto pueden interactuar en pos de contribuir a la solución de un problema tan complejo, pero a la vez tan fundamental para nuestra imagen científica del mundo.

\section{LA PROPUESTA INFORMACIONAL DE ORTIZ}

En el año 1994 aparece el primer libro de Ortiz titulado El sistema de la personalidad. Coincidentemente, en 1995, Chalmers publicaría su tesis de doctorado con el nombre de La mente consciente. En busca de una teoría fundamental. Ambos esfuerzos se caracterizan por postular la noción de información como un concepto central para resolver el problema de la conciencia. Sin embargo, son más resaltantes las diferencias entre una y otra propuesta. Ortiz (1994, 1998, 2002, 2004a, 2004b, 2008) entiende la información como una estructura material, mientras que Chalmers (1995) concibe la información como un "espacio abstracto" (p. 262), el cual no formaría parte del mundo físico. La teoría de Ortiz sería monista; en cambio, calificaría Chalmers (1995) su postura como un tipo de "dualismo naturalista" (p. 109). Otra de las diferencias centrales entre las dos perspectivas es que para Ortiz la conciencia tiene un carácter social y su estructura, correspondiente a la neocorteza cerebral, respondería al tránsito de la información, como organizador interno de la vida, hacia un tipo de organización de corte extrínseco al individuo, en una nueva faceta de la información, esta vez como información social. En términos filosóficos, esto lo ubicaría en el vértice externalista de la discusión acerca de la mente. Chalmers (1995) más bien se inclinaría por una suerte de funcionalismo no reductivo ( $\mathrm{p}$. 273), en donde la organización funcional abstracta es fundamento suficiente para postular que un sistema es consciente. Sin embargo, para el autor, la conciencia no se reduciría necesariamente a dicha organización funcional.

Una de las notorias similitudes, no obstante, está referida a entender la información como una suerte de propiedad fundamental del universo. Ambas posiciones, con matices que nos encargaremos de clarificar más adelante, encuentran en la información una suerte de criterio bisagra, que permitiría superar el vacío explicativo entre el mundo físico y la conciencia. El criterio es 
bastante diáfano: ya sea que entendamos la información como una estructura material o un criterio abstracto, postular su naturaleza fundamental lograría un tránsito fluido entre lo físico y la conciencia. Develaría el misterio de la experiencia subjetiva naturalizándola, haciéndola palpable a través de un concepto cuyo contenido semántico nos es relativamente familiar. Partamos, en primer lugar y para fines de claridad, describiendo el modelo informacional de Ortiz y cómo este autor vincula el concepto de información con la noción de conciencia; pasaremos luego a exponer el modelo infomacional de Chalmers.

Ortiz desarrolla el carácter fundamental de la información asociándola a un principio conocido: la entropía. Se sabe que este principio es la medida de desorden en un determinado sistema. Para Ortiz (1994) la información sería un proceso neguentrópico, es decir, una propiedad de organización de la materia (p. 33). Si bien la información sería un principio fundamental, este principio solo se aplicaría de forma exclusiva en el ámbito de la materia viva, sin actuar en la materia inerte, que más bien respondería no a criterios de organización, sino de mero ordenamiento. De esta forma, Ortiz (1994) considera que la información es lo que explica la unidad del ser vivo, su carácter de sistema organizado, de unidad autopermanente:

[La información es la] actividad neguentrópica de una estructura material que es la base o modelo de desarrollo de los procesos que fueron su punto de partida hasta que los convierte en el soporte funcional por medio del cual el sistema mantiene su integridad, optimiza su actividad y se reproduce a sí mismo aun en contra de los procesos entrópicos que le rodean e inciden sobre él. (p. 35)

Ortiz asume que la información es un proceso neguentrópico inherente a la vida y su organización. En otras palabras, la información se presentaría como una estructura material organizadora de la vida. Al no ser un mero criterio abstracto, como sostendría Chalmers, la información puede ser vista como una estructura material y a la vez como un proceso material. Es notorio, sin embargo, que Ortiz se refiera a este doble aspecto como tal, es decir, como un aspecto. Para el autor, nuestra condición de seres temporalmente breves determina que veamos como cambiantes procesos que duran menos que nosotros, mientras que tendamos a cosificar, a ver como cosas estables, procesos que duran más que nosotros. "Es bueno saber que este doble aspecto que atribuimos al sistema del universo es consecuencia de la forma como nos representamos el mundo en la conciencia y no la materia en sí" (Ortiz, 2008, p. 52). En consecuencia, podríamos sustentar que este enfoque le permitiría sostener un monismo metafísico de corte materialista y un dualismo, pero solo de la apariencia. En apariencia, una suerte de instrumentalismo teórico.

¿Cuál es el papel de la información con respecto a la conciencia? El autor nos invita a imaginarnos lo que ocurrió en nuestro planeta hace aproximadamente 5000 millones de años. Las moléculas se van combinando en un sentido neguentrópico, 
cada vez más organizado. A lo largo de milenios las moléculas van logrando mantener su unidad hasta que surge el ADN mitocondrial y el ADN nuclear. Una vez que la vida oficialmente inicia su camino de desarrollo, la información contenida en el ADN como proceso de reflexión material alcanza una coherencia interna a través de la coherencia con el medio que la rodea. La información genética (el ADN) codifica en el interior de la célula lo que sucede fuera de esta y se convierte en un representante relativamente autónomo del medio externo. De esta forma, el medio organiza a la célula, pero a través de la información genética que ya codificó el medio externo.

La información comienza el despliegue de la vida a través de varios niveles sucesivos; las células se convierten en tejidos, estos a su vez forman órganos, los cuales se estructuran como individuos orgánicos hasta llegar al nivel psíquico inconsciente de los animales. El carácter teleológico de la propuesta, como mencionamos previamente, radica en la afirmación de que cada nivel de desarrollo reestructura su base, es decir, el punto de inicio que generó todo el desarrollo en primer lugar. En este sentido, Ortiz (1994) identifica dos aspectos correspondientes a la actividad informacional: una actividad ascendente de tipo epigenético y una actividad descendente de tipo cinético (p. 24). Es relevante para el modelo entender que los procesos de desarrollo no son solo en una dirección, de forma mecánica. Este proceso celular alcanza, desde el nivel funcional de los órganos, un escalafón animal, correspondiente a un nivel psíquico inconsciente, hasta que una línea homínida logra producir el salto de la información como mero organizador interno hacia la información como un organizador externo, codificada en medios igualmente externos, como las pinturas que representan bisontes en una cueva.

Según Ortiz, las señales que emiten los animales se caracterizan por su carácter efímero. En contraste, los Homo sapiens logran codificar la información, que hasta el momento los había organizado internamente, en estructuras materiales fuera de ellos mismos. Esa información se constituye dada su fijeza extraindividual, dada su permanencia, como un organizador de cada uno de los individuos. Se gesta la unidad de la sociedad como un conjunto supraindividual de homínidos, organizados a partir de un nivel superior, ya no correspondiente con la inmediatez de las señales que emiten los animales, de carácter inconsciente, sino a partir de un organizador externo, fijo, permanente: la información social. Esta se convierte en formadora de la conciencia, básicamente, correspondiente con las estructuras cerebrales neocorticales:

[La conciencia es] la estructura neocortical que se organiza a partir de la actividad psíquica inconsciente del individuo humano y sobre la base de la información social, una vez que se transcribe en información psíquica consciente. Todo el conjunto de la información psíquica - afectiva, cognitiva y conativa - codificada en la memoria neocortical de las personas. (p. 309) 


\section{LA PROPUESTA INFORMACIONAL de Chalmers}

Como ya vimos, Chalmers parte de la negación del materialismo como una posible explicación de la conciencia. Se apoya, en parte, en el argumento de Jackson (1982) conocido como el $\operatorname{argu-}$ mento del conocimiento: una brillante científica llamada María es obligada a realizar sus investigaciones en un cuarto pintado únicamente en colores blanco y negro a través de un monitor, en donde solo puede ver lo que investiga también en blanco y negro. El argumento incide en el hecho de que a pesar del grado muy elevado de conocimientos que pueda obtener, aparte incluso de lo brillante que sea María, si se le permite salir del cuarto y ver las cosas en el amplio espectro cromático posible bajo condiciones normales, habrá aprendido algo más acerca de los fenómenos estudiados dentro del cuarto. Ese algo más haría que toda la explicación fisicalista que manejaba dentro del cuarto fuera insuficiente, por lo tanto, falsa. Este experimento también nos diría que la vivencia fenoménica (en este caso de color) le añadiría un dato más al modelo físico del mundo, que ya no sería abarcado por este. Trascendería las teorizaciones meramente fisicalistas.

La versión que propone el propio Chalmers (1995) consiste en el experimento mental de los zombis (p. 84). Este experimento cuestiona que la conciencia sea lógicamente superviniente en lo físico. ¿Es lógico pensar o, mejor dicho, se puede pensar sin caer en una contradicción, en la posibilidad de una versión mía, exactamente igual en el nivel microfísico, pero carente de conciencia, (un zombi)? Chalmers no se pregunta por la posibilidad empírica de un zombi, sino por la posibilidad de tener la idea de un doble zombi de este tipo sin que el pensamiento sea incoherente. Su conclusión es que sí es pensable sin incoherencia; por lo tanto, la conciencia no sería lógicamente superviniente en lo físico. Los hechos sobre la conciencia estarían más abajo o más arriba de los hechos meramente físicos (1995, p. 109). No se podrían reducir a estos últimos. Este tipo de argumentos obligan a Chalmers a buscar el principio explicativo de la experiencia fenoménica más allá (más abajo) del modelo actual del mundo físico. Como el intento de reducir las explicaciones a lo físico falla, pues lo fenoménico no es lógicamente superviniente en lo físico, existirían propiedades no físicas en el mundo. Su dualismo naturalista consiste en reconocer que existe una base material física en el mundo (una base natural), pero que las propiedades de lo consciente no están necesariamente atadas a lo físico; en otras palabras, esta base natural no agotaría los aspectos fenomenológicos de la conciencia, la cual dependería de propiedades no-físicas. El autor nos invita a pensar, por ejemplo, en el posible descubrimiento de leyes psicofísicas que descansarían sobre la postulación de propiedades más fundamentales, de un carácter más profundo, protofenoménicas. Sabemos que la física se maneja sobre propiedades fundamentales, como la masa, y que existen propiedades no fundamentales, como la temperatura. El 
descubrimiento de propiedades fundamentales protofenoménicas sería una forma de establecer un puente explicativo, entendiendo que estas protopropiedades fundamentales podrían dar cuenta de otro tipo de propiedades secundarias y derivadas, como la experiencia subjetiva (equivalente a la temperatura):

El dualismo naturalista [...] es naturalista porque propone que todo es consecuencia de una red de propiedades básicas y leyes, y porque es compatible con todos los resultados de la ciencia contemporánea [...] No se necesita que haya algo particularmente trascendente sobre la consciencia; solo es otro fenómeno natural. Todo lo que ha pasado es que nuestro cuadro de la naturaleza se ha expandido [...] Comprometerse con el dualismo no es necesariamente comprometerse con el misterio. (1995, p. 113)

Una vez asentado el panorama teórico, Chalmers (1995) propone la noción de información entendiendo, consecuentemente, que esta no formaría parte del mundo físico (p. 264). Para él, la información es un criterio formal o abstracto y puede ser entendida como un espacio informacional. Dicho espacio a su vez estaría constituido por un número de estados informacionales y una estructura básica de relaciones de diferencia entre dichos estados. Esta propuesta no se centraría en el carácter semántico o representativo de la información, sino en su carácter sintáctico, es decir, en la especificidad de un estado informacional dentro de un espacio informacional de diversas posibilidades. Para que la noción quede más clara el ejemplo que usa el autor es el siguiente: el espacio más simple que se puede representar sería el consistente en dos estados y una diferencia primitiva entre uno y otro. Estos dos estados podrían ser entendidos como dos bits de información, 0 y 1. El hecho de que estos dos estados sean diferentes el uno del otro agota su naturaleza. Es decir, este espacio informacional está caracterizado completamente por su estructura de diferencias.

Los espacios informacionales, obviamente, pueden ser más complejos y no solo estar representados por números discretos, sino también por magnitudes continuas como las cifras infinitas que se encuentran entre el cero y el uno. Asimismo, pueden representarse como espacios multidimensionales, análogos a regiones igualmente multidimensionales. Lo relevante es la realización física de estos espacios. Para el autor no es difícil distinguir dicho fenómeno. Por ejemplo, el interruptor de la luz sería un sistema físico que realizaría un espacio informacional similar al utilizado como ejemplo en el párrafo precedente. La información sería una diferencia que hace la diferencia, es decir, para que la luz se encienda la única diferencia que le "interesaría" al interruptor sería la diferencia entre prendido (1) y apagado (0). Con este ejemplo, Chalmers da a entender que podría asumirse un camino causal que ocurriría gracias a la realización física de la información como espacio informacional, consistente en estados informacionales cuya estructura responde a las diferencias entre uno y otro estado.

Para el caso de las experiencias fenoménicas, Chalmers (1995) apela a su principio de doble aspecto. Según el 
principio, "cuando encontramos un espacio informacional realizado fenoménicamente, encontramos este mismo espacio informacional realizado físicamente" (p. 267). Dada esta realización física, podrían generarse hipótesis que hicieran corresponder áreas de la corteza cerebral con cada una de estas realizaciones. ¿Cómo la información se realiza fenoménicamente? Habíamos señalado previamente que los espacios informacionales pueden tener una complejidad incrementada, incluso con estructuras de diferencias que respondan a espacios informacionales de tres dimensiones. Para el caso, por ejemplo, de la experiencia visual que realiza un espacio de este tipo, esta realización de un espacio informacional, según Chalmers, correspondería con un espacio tridimensional de representaciones codificadas neuralmente en la corteza visual. Los elementos de este espacio tridimensional se relacionarían directamente con elementos del espacio informacional fenoménico.

\section{DISCUSIÓN}

A partir de lo expuesto, podríamos intentar distinguir qué tipos de preguntas responde cada una de las teorías y hasta qué punto podríamos establecer relaciones entre una y otra. Si nos centramos en el vacío explicativo, podemos afirmar que la teoría de Ortiz logra explicar la transición de lo físico a lo consciente a través de la materialización de la información, así como el planteamiento teleológico (cinético) de los procesos de desarrollo. La idea de la información como una estructura material es coherente con la idea de que dicha estructura puede a su vez estructurar la neocorteza cerebral y de esta forma constituir la conciencia, que no sería otra cosa que dicha corteza reestructurada por acción de la información social. Como el proceso del desarrollo de la información es doble, una vez que las personas produjeron la información social fuera de ellas mismas, esta modificaría sus bases iniciales de desarrollo (las personas) convirtiéndolas en el soporte consciente de la estructura de la sociedad. El modelo también daría cuenta de una intuición bastante común: de alguna manera, la información que recibimos de la sociedad es lo que nos hace conscientes.

Lo que se mostraría como problemático para preservar el carácter fenoménico de la conciencia es el enfoque aparentemente instrumentalista de la propuesta. Si el doble aspecto de la información solo aparece como un fenómeno producto de nuestra forma de ver procesos de corta o larga duración, este criterio se podría extender a la experiencia subjetiva. El razonamiento sería el siguiente: dado que los pensamientos tienen un carácter claramente episódico, es decir, de menor duración que nosotros mismos, se nos aparecerían como una actividad, pero en realidad no serían otra cosa más que estructuras neuronales. Estaríamos frente a un tipo de, en el mejor de los casos, reduccionismo de la conciencia fenoménica y, en el peor de los casos, una simple y llana eliminación de la misma. La experiencia subjetiva sería mera apariencia de actividad. No obstante, es posible una interpretación alternativa del modelo, si se entiende a la conciencia 
fenoménica como igual de duradera que nosotros mismos (recordemos el principio de ineluctabilidad). Al ser indistinguible de nosotros mismos, dada su presencia permanente, no tendría que ser concebida como mera apariencia de actividad.

¿Cómo conciliar la discrepancia entre los dos modelos con relación a la materialidad de la información? Es bueno notar que Chalmers proviene del ámbito de las matemáticas; esto se deja ver en su elección del modelo informacional de Shannon (básicamente un modelo matemático) y en su acercamiento individualista a la conciencia. En ninguna parte en su modelo, aparece la inclusión del factor social como un elemento relevante para explicar la conciencia. Esto también explica su inclinación por entender a la información como una propiedad fundamental del universo y su aplicación directa al problema de la conciencia. Según Seager (2016), aparecerían dos problemas en la elección de un enfoque de este tipo (pp. 292-293). El primero estaría referido a la irrelevancia causal producida por entender la conciencia con base en una descripción funcional. Dado que toda descripción funcional debe ser instanciada en lo físico, lo que haría el verdadero trabajo causal sería justamente lo físico. La conciencia se vería reducida a un epifenómeno sin relevancia causal de ningún tipo. En segundo lugar, si se postulase la conciencia (producto de la realización física de espacios informacionales) como una propiedad fundamental del universo (tal como la masa, por ejemplo), ¿cómo podríamos justificar su carácter fundamental si solo puede tener plausibilidad causal instanciada en una estructura física? El modelo de Chalmers no podría evitar regresar a depender de una ontología fisicalista clásica, cosa que, como vimos, quería impedir desde el inicio.

Viéndolo con mayor precisión, Ortiz se aleja de entender la información como una propiedad fundamental del universo cuando interpreta dicha información como un proceso exclusivo de la vida. Esta suerte de estipulación proviene de la necesidad de establecer una explicación de la vida humana, para lo cual Ortiz encuentra que la conversión de simple información vital en información social le permite explicar el salto del animal inconsciente a la persona consciente. Sin embargo, es interesante notar que una de las fuentes que le permite postular su teoría discrepa con esta visión particularista de la información:

La información como tal tampoco surge nunca; al parecer también es una propiedad tan inseparable de la materia como el espacio, el tiempo, el movimiento, etc. Sin embargo, podemos hablar del surgimiento de la capacidad de utilizar información, es decir, del control. El utilizar la información (y a partir de ello también propiedades suyas como el valor y después el sentido) nace realmente por primera vez al aparecer los seres vivos como sistemas cibernéticos genéticamente primarios. (Ursul, 1972, p. 121)

Como se puede apreciar, ambas propuestas señalan caminos posibles de solución frente al difícil problema de la conciencia. 


\section{RefERENCIAS}

Chalmers, D. (1995). The Conscious Mind: In Search of a Fundamental Theory. Recuperado de http:/consc.net/.

Gabriel, M. (2016). Yo no soy mi cerebro. Filosofía de la mente para el siglo XXI. Barcelona: Pasado y Presente.

Kriegel, U. (Ed.). (2014). Current Controversies in Philosophy of Mind. Nueva York: Routledge.

Kuhn, T. (2006). La estructura de las revoluciones cientificas. México D. F.: Fondo de Cultura Económica.

Ortiz, P. (1994). El sistema de la personalidad. Lima: Centro Gráfico Orión.

Ortiz, P. (1997). La formación de la personalidad. Algunos aspectos de interés pedagógico. Lima: Secretaría de Cultura del Colegio de Doctores en Educación del Perú.

Ortiz, P. (2002). Lenguaje y habla personal. Lima: Fondo Editorial, Universidad Nacional Mayor de San Marcos.

Ortiz, P. (2004a). Cuadernos de psicobiología social 1. Introducción a una psicobiología del hombre. Lima: Fondo Editorial, Universidad Nacional Mayor de San Marcos.
Ortiz, P. (2004b). Cuadernos de psicobiología social 6. El nivel consciente de la actividad personal. Lima: Fondo Editorial, Universidad Nacional Mayor de San Marcos.

Ortiz, P. (2008). Educación y formación de la personalidad. Lima: Fondo Editorial de la Universidad de Ciencias y Humanidades.

Popper, K. (1994). Conjeturas y refutaciones. Barcelona: Paidós.

Putnam, H. (1981) Reason, Truth and History. Cambridge: Cambridge University Press.

Putnam, H. (1994). Reductionism and the Nature of Psychology. En J. Conant (Ed.), Words and Life (pp. 428-441). Cambridge: Harvard University Press.

Seager, W. (2016). Theories of Consciousness. An Introduction and Assessment. Nueva York: Routledge.

Siewert, Ch. (2014). Speaking Up for Consciousness. En U. Kriegel (Ed.), Current Controversies in Philosophy of Mind (pp. 199-221). Nueva York: Routledge.

Ursul, A. D. (1972). Naturaleza de la información. Montevideo: Ediciones Pueblos Unidos. 